\title{
Variabilité Temporelle Des Températures Annuelles Et Saisonnières Dans l'Est Algérien
}

\author{
Laala Ahmed, Ma \\ Alatou Djamel, Pr
}

Laboratoire de Développement et Valorisation des Ressources

Phytogénétiques, Département de Biologie et Ecologie Végétale, Université des Frères Mentouri Constantin, Algérie

\section{doi: 10.19044/esj.2016.v12n14p45 URL:http://dx.doi.org/10.19044/esj.2016.v12n14p45}

\begin{abstract}
In this study, we tried to characterize the tendency of some thermal variables and their annual fluctuations over a period of 30 years (1982-2011). The results show a spatial and temporal variability of the anomaly to the minimum and maximum temperatures. Cold years represent a percentage ranging from 40 to $57 \%$ of the years studied, while hot are estimated at $63 \%$ for the years analyzed. Thus, the analysis of long series of minimum and maximum temperature reveals statistically significant trends which present spatial variability for annual and spatiotemporal temperatures to seasonal temperatures. An evolution of annual maximum temperatures to a warming has been recorded for the majority of the provinces of eastern Algeria with notable increases up to $+3^{\circ} \mathrm{C} / 30$ years for the wilaya of Batna. However, the minimum temperatures have downward trends in the range of $-1.5^{\circ} \mathrm{C}$ and $2^{\circ} \mathrm{C} / 30$ years respectively in Jijel and Setif and upward trends varying between 0.5 and $1.7^{\circ} \mathrm{C} / 30$ years. After this study, calculates the thermal anomaly and mapping trends can create a regional picture of the evolution of the temperature in eastern Algeria that shows a significant warming of this region over the past decades especially for the wilaya of Batna. This study includes a reference to define strategies to adapt to thermal variability in eastern Algeria.
\end{abstract}

Keywords: Variability, temperature, Trend, Anomaly, eastern Algeria

\section{Résumé}

Dans cette étude, on a essayé de caractériser la tendance de quelques variables thermiques et leurs fluctuations annuelles sur une période de 30 ans (1982-2011). Les résultats obtenus montrent une variabilité spatio-temporelle de l'anomalie pour les températures minimales et maximales. Les années 
froides représentent un pourcentage variant de 40 à 57\% des années étudiées, alors que les chaudes sont estimées à 63\% pour les années analysées. Ainsi, l'analyse de longues séries de température minimale et maximale révèle l'existence des tendances statistiquement significatives qui présentent une variabilité spatiale pour les températures annuelles et spatiotemporelle pour les températures saisonnières. Une évolution des températures maximales annuelles vers un réchauffement a été enregistré pour la majorité des wilayas de l'Est algérien avec des hausses remarquables pouvant atteindre $+3^{\circ} \mathrm{C} / 30$ ans pour la wilaya de Batna. Cependant, les températures minimales présentent des tendances à la baisse de l'ordre de $-1.5^{\circ} \mathrm{C} / 30$ ans et $-2^{\circ} \mathrm{C} / 30$ ans respectivement pour la wilaya de Jijel et Sétif et des tendances à la hausse variant entre 0.5 et $1.7^{\circ} \mathrm{C} / 30 \mathrm{ans}$. Au terme de cette étude, le calcule de l'anomalie thermique et la cartographie des tendances permettent de créer une image régionale de l'évolution de la température à l'Est algérien qui montre un réchauffement notable de cette région au cours de ces dernières décennies surtout pour la wilaya de Batna. Cette étude constitue aussi une référence pour définir des stratégies d'adaptation face à la variabilité thermique au niveau de l'Est algérien.

Mots clés : Variabilité, Température, Tendance, Anomalie, Est algérien

\section{Introduction}

La température est un facteur limitant qui conditionne pour une large part la distribution des végétaux et peut déterminer leur productivité. C'est un paramètre qui varie dans l'espace et dans le temps. Elle a des effets directs sur plusieurs processus vitaux de la plante tels que la photosynthèse, les mouvements de solutés dans les tissus spécialisés, la respiration et la croissance (Berry et Bjorkman 1980, Havaux et Lannoye 1985, Méthy et Trabaud 1990, King et al.,2006, Myneni et al.,1997). Ainsi, Heller et al., (1989) ont montré que l'effet de la température sur la croissance est la résultante de son action sur la photosynthèse et les réactions métaboliques ainsi que sur l'alimentation hydrique et minérale.

Ce sont les températures extrêmes qui ont une influence sur la végétation, sauf si elles sont exceptionnelles et de courte durée (Dajoz, 1985). Certaines études prévoient que la température pourrait également influencer les aires de répartition des écosystèmes forestiers (Haman et Wang 2006; McKenney et al., 2007). Avec le réchauffement de la terre, les espèces tendent à déplacer leurs aires vers des latitudes et des altitudes plus élevées (Davis, 1989). L'étude de la fluctuation de la température et de son évolution est considérée comme une étape clé pour prédire les régions exposées au risque de réchauffement ou refroidissement et par conséquence aider les décideurs à la mise en place d'une stratégie d'adaptation régionale 
ou nationale pour la protection des essences forestières. Dans cette optique, la présente étude vise à analyser la variabilité interannuelle des températures minimales et maximales et à cartographier particulièrement la tendance de ces paramètres dans le Nord Est algérien, région qui renferme la quasitotalité des forêts algériennes.

\section{Présentation de la zone d'étude}

La zone d'étude est localisée dans la partie Nord-Est du territoire algérien (Fig.1) comprise entre les latitudes 34 $31^{\prime} 15^{\prime \prime}$ et $37^{\circ} 5^{\prime} 24^{\prime \prime}$ Nord et les longitudes $4^{\circ} 19^{\prime} 57^{\prime \prime}$ et $8^{\circ} 41^{\prime} 2^{\prime \prime}$ Est, limitée à l’Est par la frontière Algéro-Tunisienne, à l'Ouest par la vallée d'Oued Soummam et les monts des Bibans, au Sud par le piémont Sud de l'Atlas Saharien et au Nord par la rive Sud de la mer méditerranéenne.

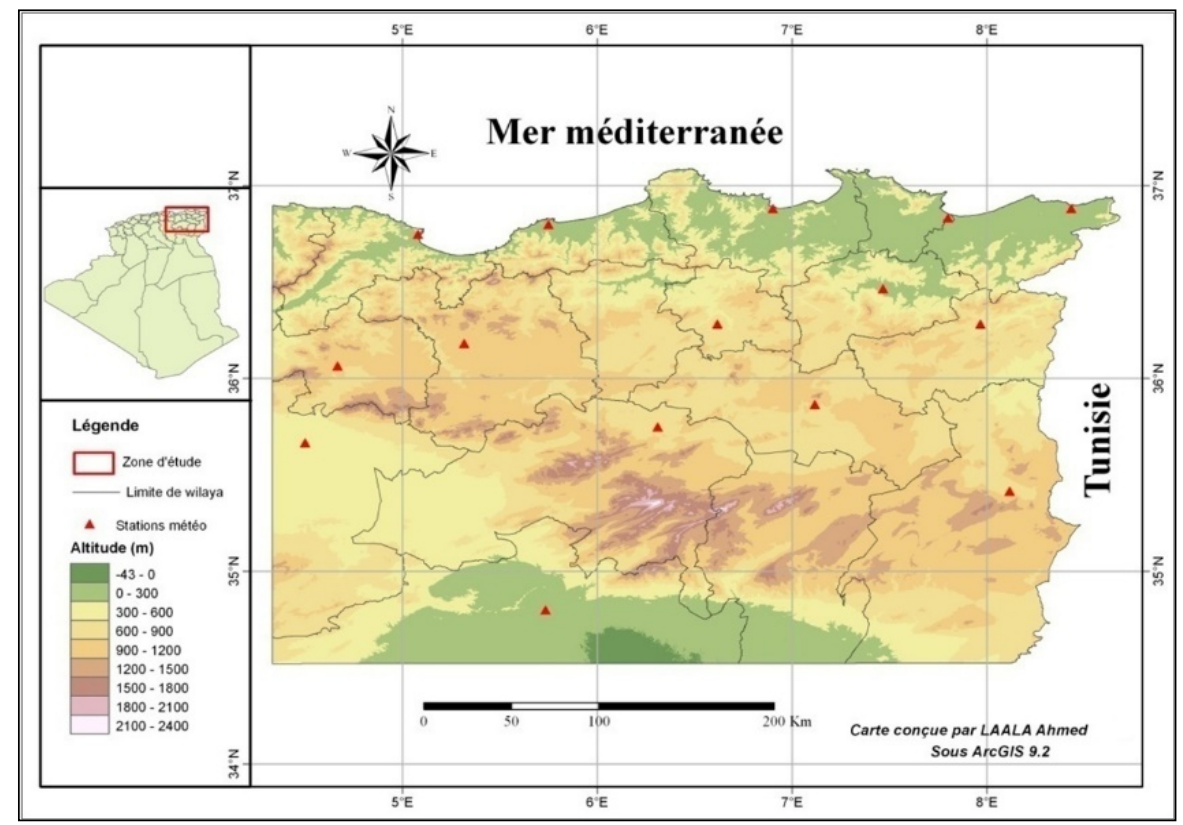

Fig.1 : Localisation et énergie du relief

\section{Matériels et méthodes}

\section{Données utilisées}

Les données thermiques mises à notre disposition proviennent des stations météorologiques pour une période de 30 ans (1982-2011). Selon le Groupe d'experts intergouvernemental sur l'évolution du climat (GIEC, 2007), cette période est suffisamment longue pour être significative de la variabilité et la fluctuation de la température et pas de leur évolution à long terme (changement climatique). 15 stations météorologiques ont été retenues, fournissant des données thermiques complètes par rapport à d'autres stations qui possèdent des données lacunaires ou manquantes. 


\section{Matériel}

Tous les traitements statistiques ont été effectués par le biais du logiciel XLSTAT 2011, alors que la création des cartes a été réalisée au moyen du logiciel ArcGIS 9.2.

\section{Méthodes de traitement des données}

Pour les calculs saisonniers, l’hiver a été défini comme la période de décembre de l'année n -1 à janvier et février de l'année n; le printemps regroupe les mois de mars à mai; l'été les mois de juin à août et l'automne la période de septembre à novembre.

\section{A- Lissage par moyennes mobiles}

Pour supprimer les fluctuations transitoires, on a lissé les valeurs de la série statistique par l'utilisation des moyennes mobiles (glissantes) calculées sur une période de 3 ans de façon à souligner les tendances à long terme des températures annuelles et saisonniers.

\section{B- Estimation de l'anomalie des températures}

L’anomalie climatique est la déviation par rapport à un état climatique moyen (Merle, 1980). L'estimation de ce paramètre est calculée par la comparaison des températures annuelles avec celles calculées sur la période de référence (1982-2011) pour mieux distinguer les années exceptionnellement froides et chaudes.

Nous avons considéré qu'une année était froide, lorsque l'anomalie prend des valeurs négatives, c'est-à-dire que la moyenne annuelle est inférieure à la moyenne des températures calculée sur la période (19822011). Au contraire, l’anomalie est positive pour les périodes chaudes où la moyenne annuelle dépasse la moyenne du 30 années analysées.

Ainsi, pour déterminer l'intensité des années froides et chaudes, nous avons choisi les paramètres suivants :

- Le nombre d'années froides ou chaudes ;

- Le poids $\left(\mathrm{en}^{\circ} \mathrm{C}\right)$ : représente la somme des valeurs de l'anomalie pour les années froides ou chaudes: par exemple : si la moyenne des températures minimales de la période de 30 ans est égale à $12^{\circ} \mathrm{C}$, une année caractérisé par une température minimale de $11.5^{\circ} \mathrm{C}$ aura un poids de : $\left(11.5^{\circ} \mathrm{C}-12^{\circ} \mathrm{C}\right)=-0.5^{\circ} \mathrm{C}$. On fait la somme de ces valeurs pour toute la durée considéré comme froide ;

- L’intensité: (en ${ }^{\circ} \mathrm{C} / \mathrm{an}$ ) : représente le poids par rapport à la durée : des périodes froides peuvent être longues et modérées, d’autres courtes mais intenses. Ce paramètre nous permettra d'avoir un indicateur intéressant à ce niveau. 


\section{C- Estimation de la tendance}

La tendance est l'orientation générale d'une série d'observations à la hausse ou à la baisse sur une période assez longue. Dans notre cas, le calcul de la tendance a été estimé avec la méthode des moindres carrés pour ajuster la série chronologique.

On détermine la droite des moindres carrés $(\mathrm{y}=\mathrm{ax}+\mathrm{b})$ du nuage de points $(\mathrm{X}, \mathrm{Y})$, c'est-à-dire la droite qui minimise la distance:

$$
\text { D'où : } \quad a=\operatorname{cov}(x, y) / X^{2} \quad \text { et } \quad b=m(y)-a m(x)
$$

La tendance est obtenue par le calcul de la pente $a$ qui est un coefficient directeur :

- Si a $>0$, on a une tendance à la hausse ;

- Si a $<0$, on a une tendance à la baisse

Le niveau significatif ( $\alpha=5 \%$ ) de la tendance a été estimé selon la méthode Mann-Kendall non paramétrisée (Mann, 1945; Kendall, 1975). Les résultats obtenus sont représentés sous forme cartographique. Les tendances positives et significatives à 5\% sont représentées par un triangle de couleur rouge orienté vers le haut, alors que les tendances négatives et significatives à $5 \%$ sont représentées par un triangle bleu orienté vers le bas. Les tendances non significatives sont affichées par un triangle noir. La taille des triangles est proportionnelle à l'intensité de la tendance.

\section{Résultats}

\section{Variabilité interannuelle des températures minimales :}

L'anomalie calculée pour les températures minimales varie en fonction de l'année analysée et de la wilaya concernée. La plus forte anomalie négative est enregistrée à la wilaya de Msila durant l'année 2005, alors que celle positive a été affichée au niveau de la wilaya de Constantine durant l'année 1997 (fig.2).

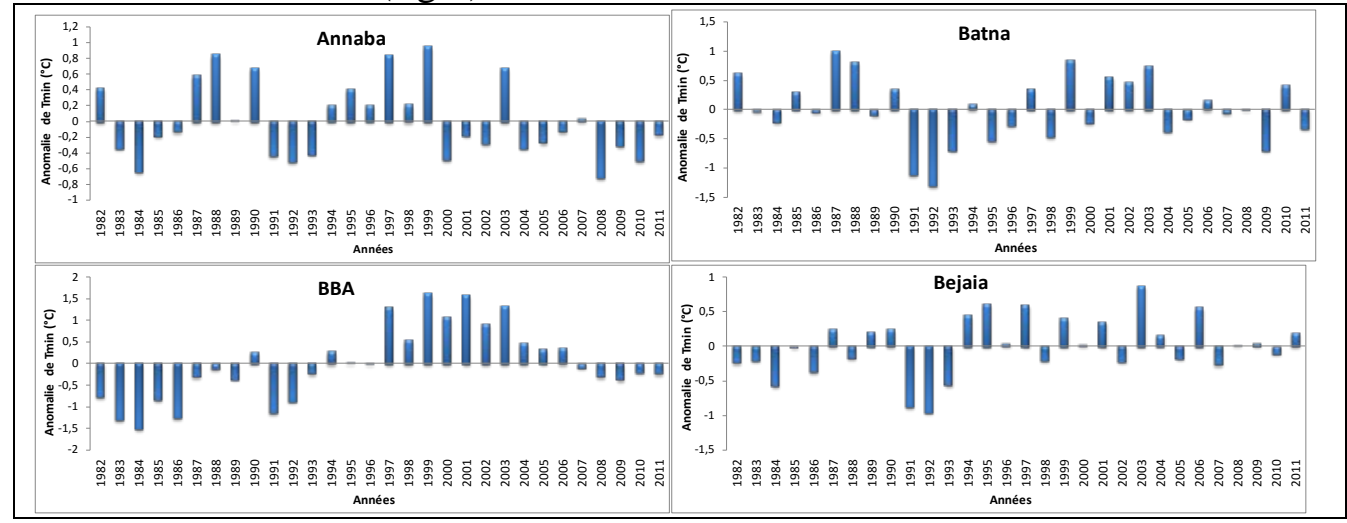




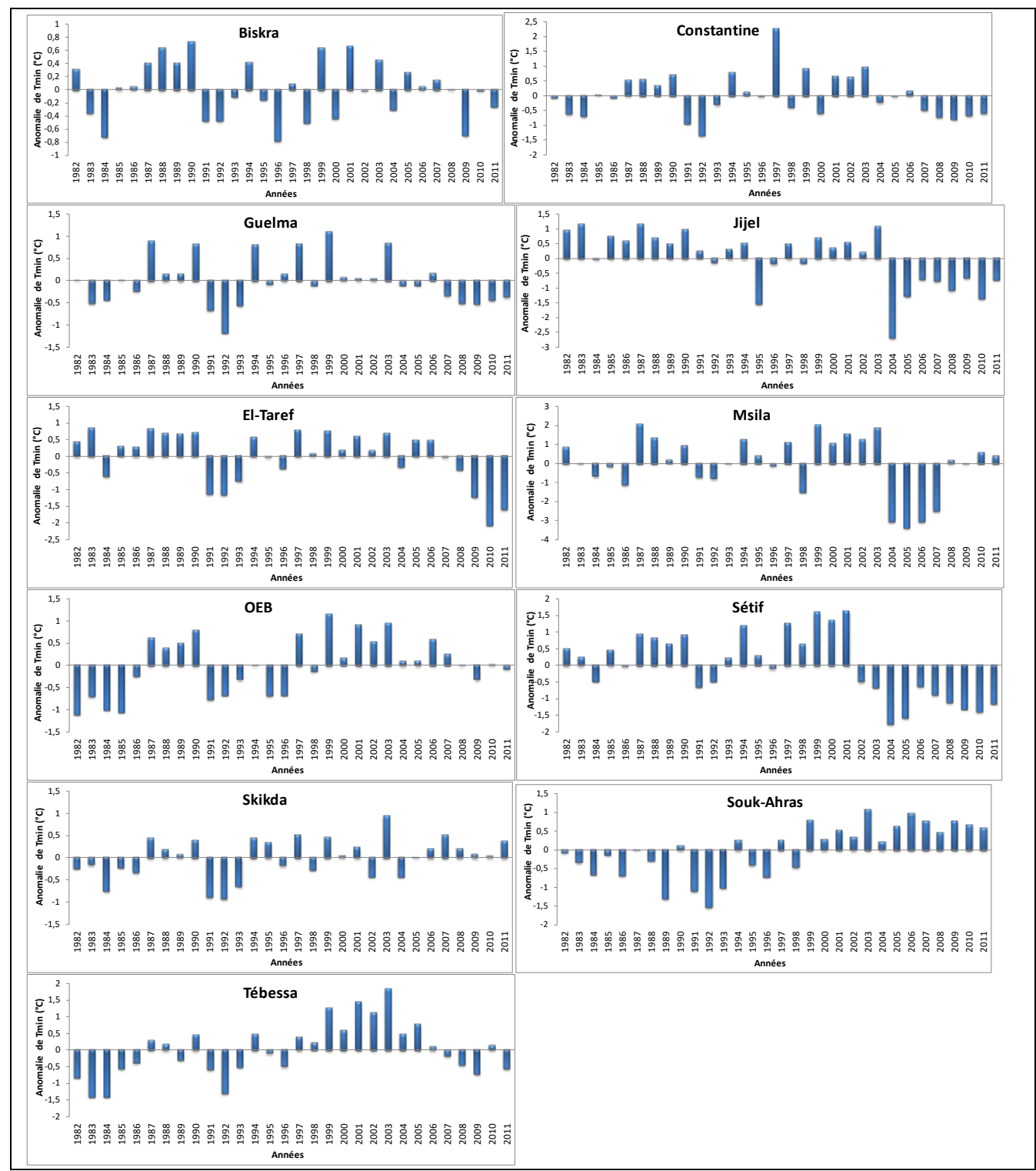

Fig.2 : Anomalie des températures minimales au cours de la période 1982-2011

Le nombre des années froides fluctue entre 12 et 17 années avec un maximum enregistré au niveau de la wilaya d'Annaba, Constantine, Bordj Bou-Arreridj, Batna et un minimum affiché dans la wilaya d’El-Taref et Msila. On remarque également que les wilayas de Bordj Bou-Arreridj, Constantine, El-Taref et Guelma ont connu une période froide durant cinq ans consécutives (2007-2011) où les températures annuelles minimales sont inferieure à la normale calculé sur la période de 30 ans. Ainsi, la période 1982-1986 est une période froide pour Bejaia, Souk-Ahras, Tbessa, Bordj Bou-Arreridj, Skikda et Oum El Bouaghi (fig.2). 


\section{Variabilité interannuelle des températures maximales}

L'histogramme d'anomalie des températures maximales annuelles reflète bien la grande variabilité interannuelle de ce paramètre dans l'Est algérien (fig.3) qui est basée sur les fluctuations entre les années froides et les années chaudes. Selon la wilaya étudiée, le nombre des années chaudes varie de 14 à 19 années. La période 2005-2011 est une période chaude pour la wilaya de Batna, Constantine, Sétif, Bordj Bou-Arreridj, Biskra, Msila, Skikda, Guelma et Jijel où les températures annuelles maximales peuvent gagner $1.9^{\circ} \mathrm{C}$, ce qui nous indique le réchauffement notable de ces régions durant les sept dernières années de la période d'étude.

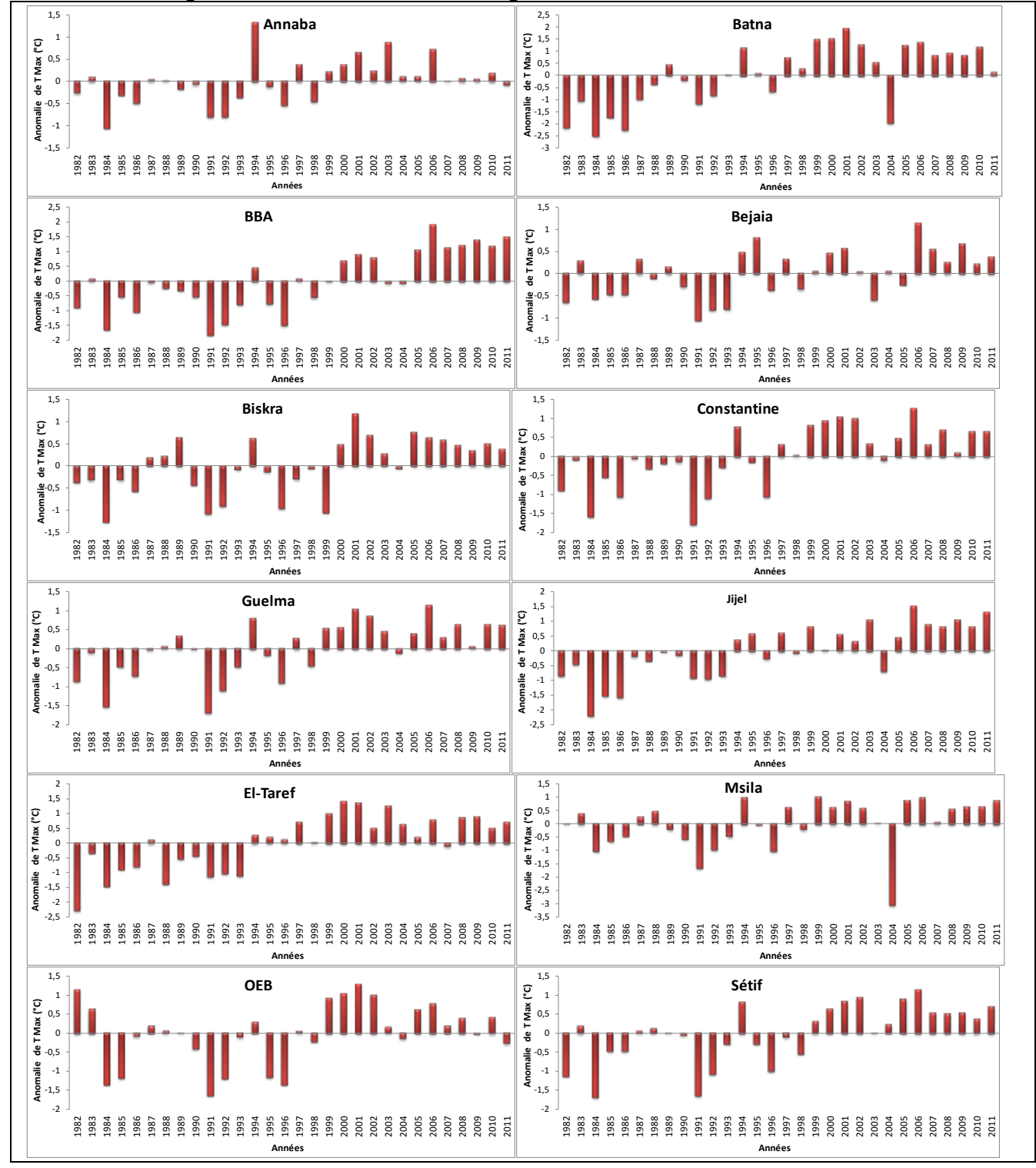




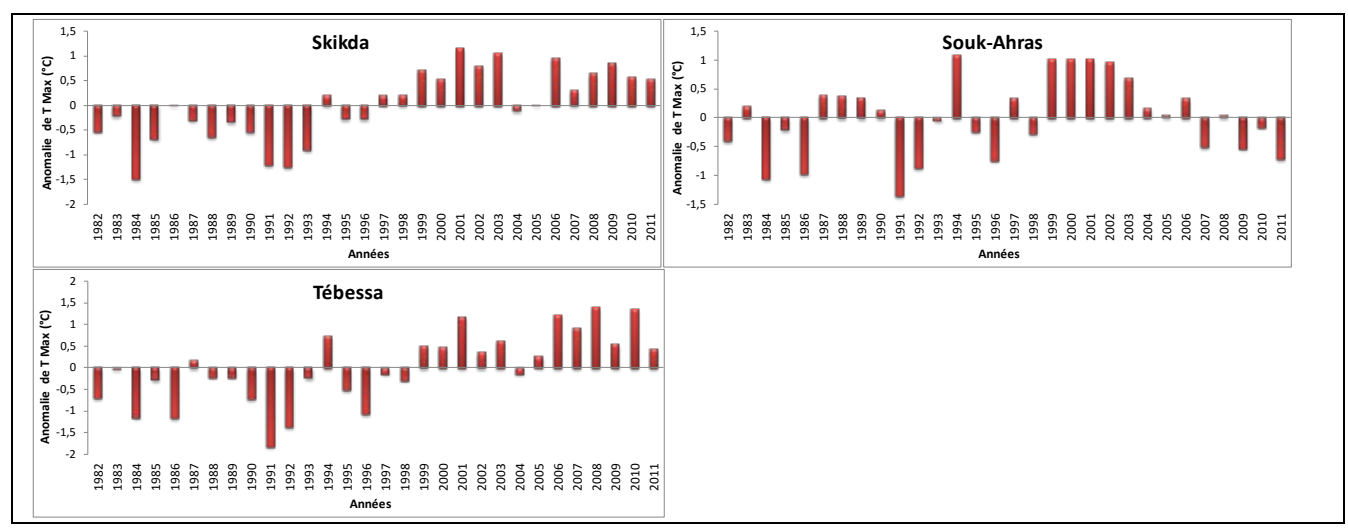

Fig.3 : Anomalie des températures maximales au cours de la période 1982-2011

La figure $\mathrm{n}^{\circ} 4$ illustre l'intensité des années froides et chaudes pour la période 1982-2011. La taille des sphères est proportionnelle à l'intensité de la période froide ou chaude : plus les sphères sont petites plus l'intensité est faible.

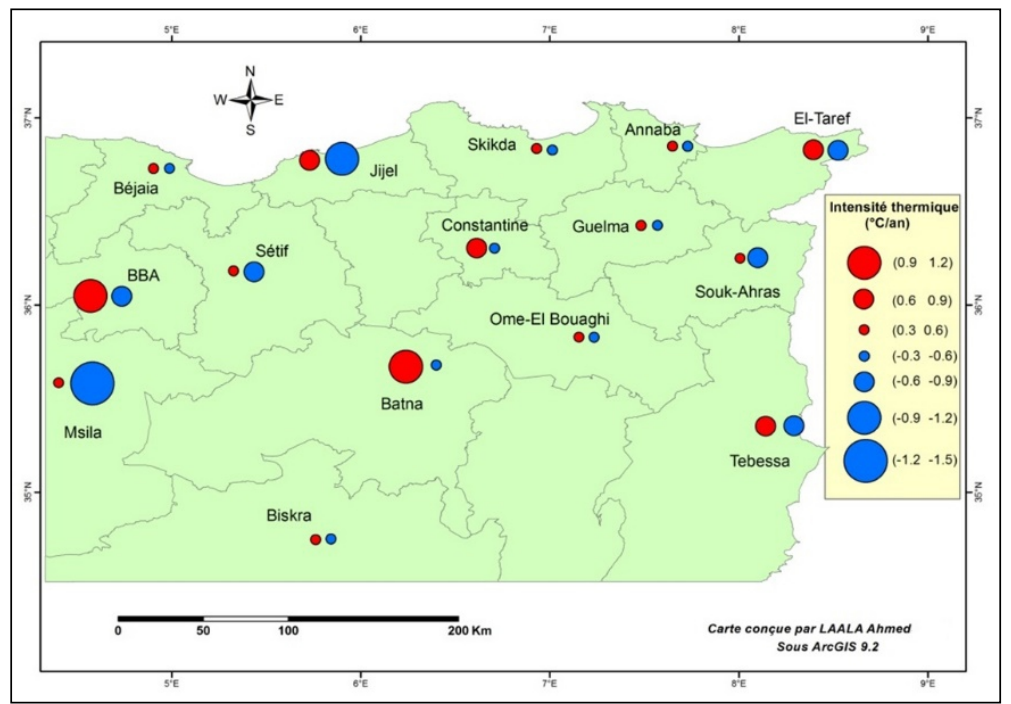

Fig.4 : Intensité des années froides et chaudes calculée sur la période 1982-2011

Sur la base du graphique ci-dessus, on remarque que:

Six wilayas de l'Est algérien affichent des périodes de réchauffement d'une intensité supérieur à $0.6^{\circ} \mathrm{C} / \mathrm{an}$. Celles de Batna et Bordj Bou-Arreridj présentent des périodes chaudes très intenses $\left(0.9^{\circ} \mathrm{C} / \mathrm{an}\right)$, avec un poids thermique respectivement de l'ordre de $15.8^{\circ} \mathrm{C}$ et $12.2^{\circ} \mathrm{C}$. Le reste des wilayas marque des périodes chaudes avec une intensité comprise entre 0.3 et $0.6^{\circ} \mathrm{C} / \mathrm{an}$ (fig.4).

Il apparaît aussi que la période froide la plus intense $\left(1.4^{\circ} \mathrm{C} / \mathrm{an}\right)$ est enregistrée au niveau de la wilaya de Msila, elle est tout à fait exceptionnelle 
par sa durée (12 ans) et son poids $\left(-16.9^{\circ} \mathrm{C}\right)$. La wilaya de Jijel marque aussi une période froide avec un poids égal à $-11.2^{\circ} \mathrm{C}$ enregistrée durant 13 ans, soit une intensité de $-0.9^{\circ} \mathrm{C} / \mathrm{an}$. Les autres wilayas affichent des périodes de refroidissement d'une intensité moins importante $\left(<-0.9^{\circ} \mathrm{C} / \mathrm{an}\right)$.

Il faut également constater que malgré la durée remarquable des années froides enregistrée à la wilaya d'Annaba (17ans), elle apparaît peut intense $\left(-0.4^{\circ} \mathrm{C} /\right.$ ans $)$ avec un poids relativement faible $\left(-6.1^{\circ} \mathrm{C}\right)$.

\section{Tendances des températures annuelles}

On a essayé également d'analyser les tendances thermiques (annuelles et saisonnières) sur une période de 30 ans (1982-2011). L’unité de ces tendances est de ${ }^{\circ} \mathrm{C} / 30$ ans.

\section{A)- Température minimale annuelle}

On remarque une variabilité spatiale de la tendance des températures minimales annuelles calculé pour la période 1982-2011 (fig.5). Parmi les 15 séries analysées, on a 8 séries des températures minimales annuelles qui affichent des tendances statistiquement significatives au seuil de $5 \%$.

Concernant les tendances significativement positives, elles sont observées au niveau de 5 wilayas (Bordj Bou-Arreridj, Souk-Ahras, Tébessa, Oum El-Bouaghi et Skikda). La tendance maximale est affichée à la wilaya de Bordj Bou-Arreridj $\left(1.7^{\circ} \mathrm{C} / 30\right.$ ans), alors que la minimale est enregistrée pour la wilaya de Skikda $\left(0.5^{\circ} \mathrm{C} / 30\right.$ ans $)$. Les tendances significativement négatives sont affichées uniquement au niveau de trois wilayas (El-Taref, Sétif et Jijel) avec des tendances respectivement de $-0.8^{\circ} \mathrm{C} / 30,-1.5^{\circ} \mathrm{C} / 30$ et $2^{\circ} \mathrm{C} / 30$.

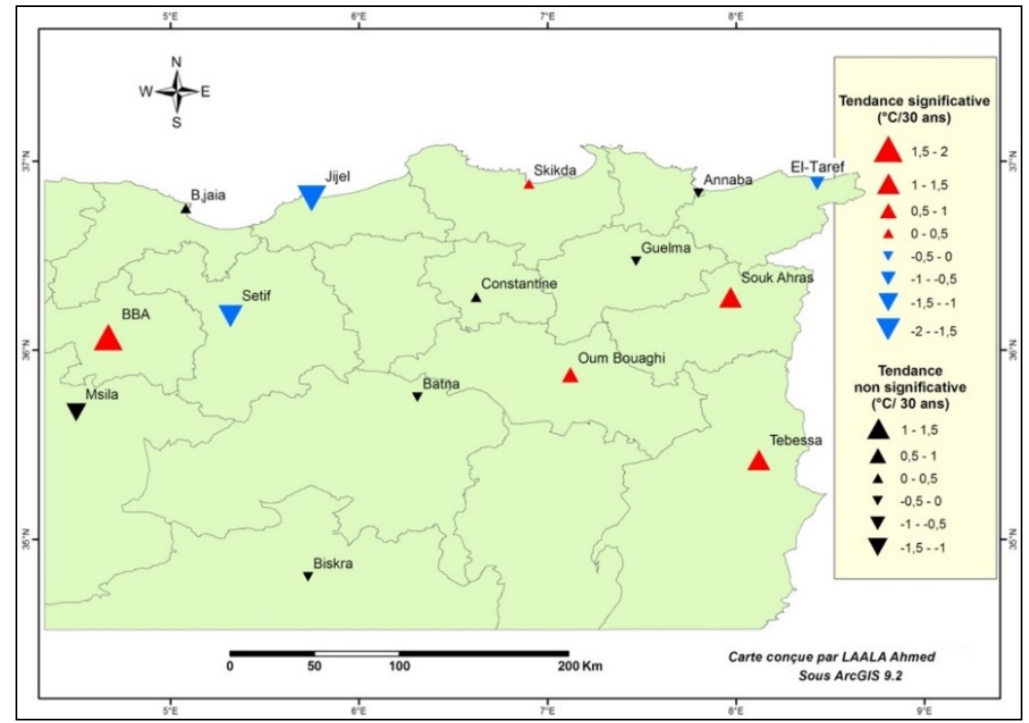

Fig.5: Tendances des températures minimales annuelles (1982-2011) 


\section{B)- Température maximale annuelle}

Un réchauffement remarquable a été enregistré dans toutes les wilayas de l'Est algérien où les tendances des températures maximales annuelles, calculées sur la période (1982-2011), sont toutes à la hausse (fig.6). L'intensité de réchauffement varie spatialement d'une wilaya à l'autre. Il fluctue entre $0.7^{\circ} \mathrm{C} / 30$ ans au niveau d'Annaba à $3.03^{\circ} \mathrm{C} / 30$ ans pour la wilaya de Batna. Les tendances non significatives sont affichées uniquement au niveau de deux wilayas: Souk-Ahras et Oum-El Bouaghi.

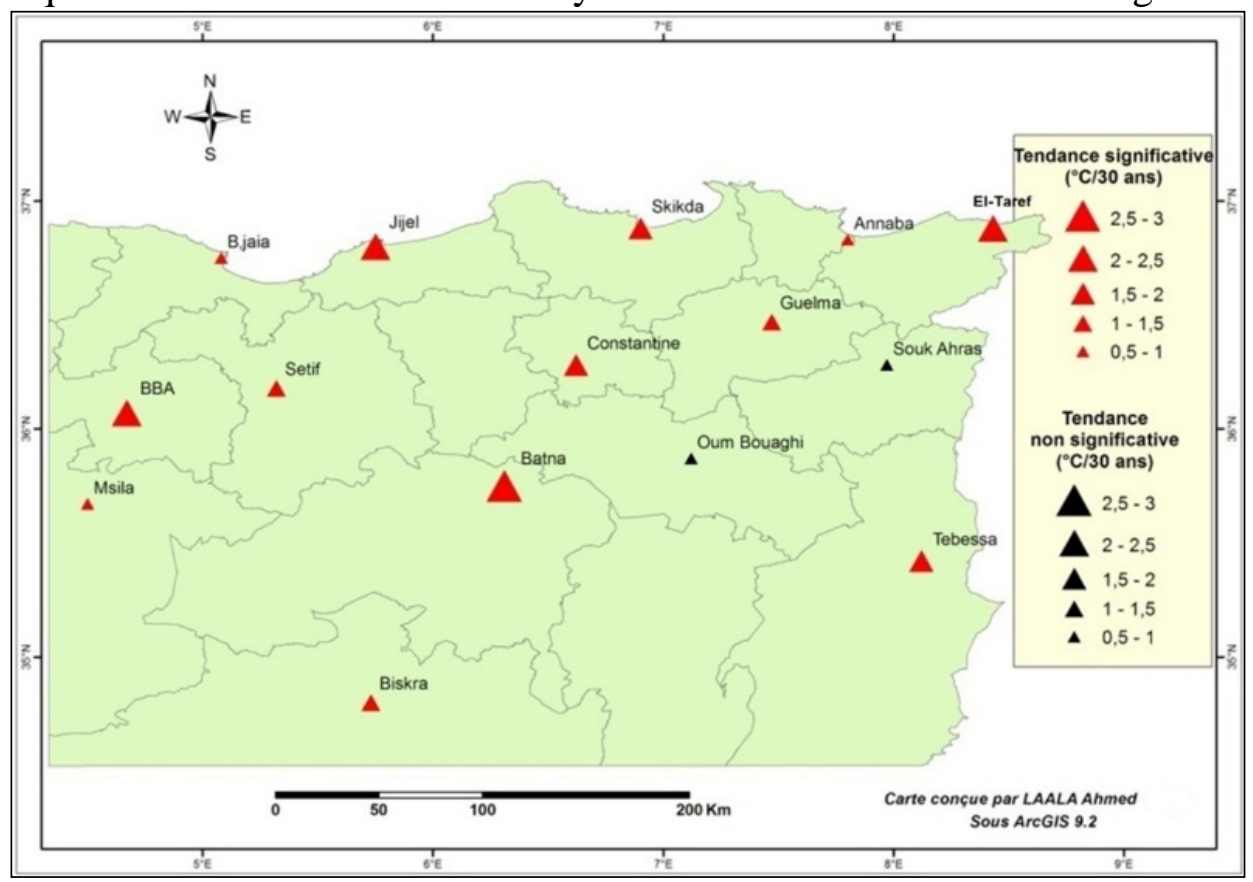

Fig.6: Tendances des températures maximales annuelles (1982-2011)

La lecture de la carte $n^{\circ} 5$ et 6 , nous montre que les températures maximales annuelle connaissent une hausse plus prononcé que les températures minimales annuelles et cela durant la période étudiée 19822011.

\section{C)- L'amplitude thermique annuelle}

Le paramètre de l'amplitude thermique annuelle marque une hausse dans la majorité des wilayas avec une valeur minimale de $0.13^{\circ} \mathrm{C} / 30$ ans enregistrée à Tébessa et une valeur maximale de $4.2^{\circ} \mathrm{C} / 30$ ans affichées au niveau de Jijel. Cependant Souk-Ahras est la seule station qui présente une baisse significative de l'amplitude thermique annuelle $\left(-1^{\circ} \mathrm{C} / 30\right.$ ans) (fig.7). 


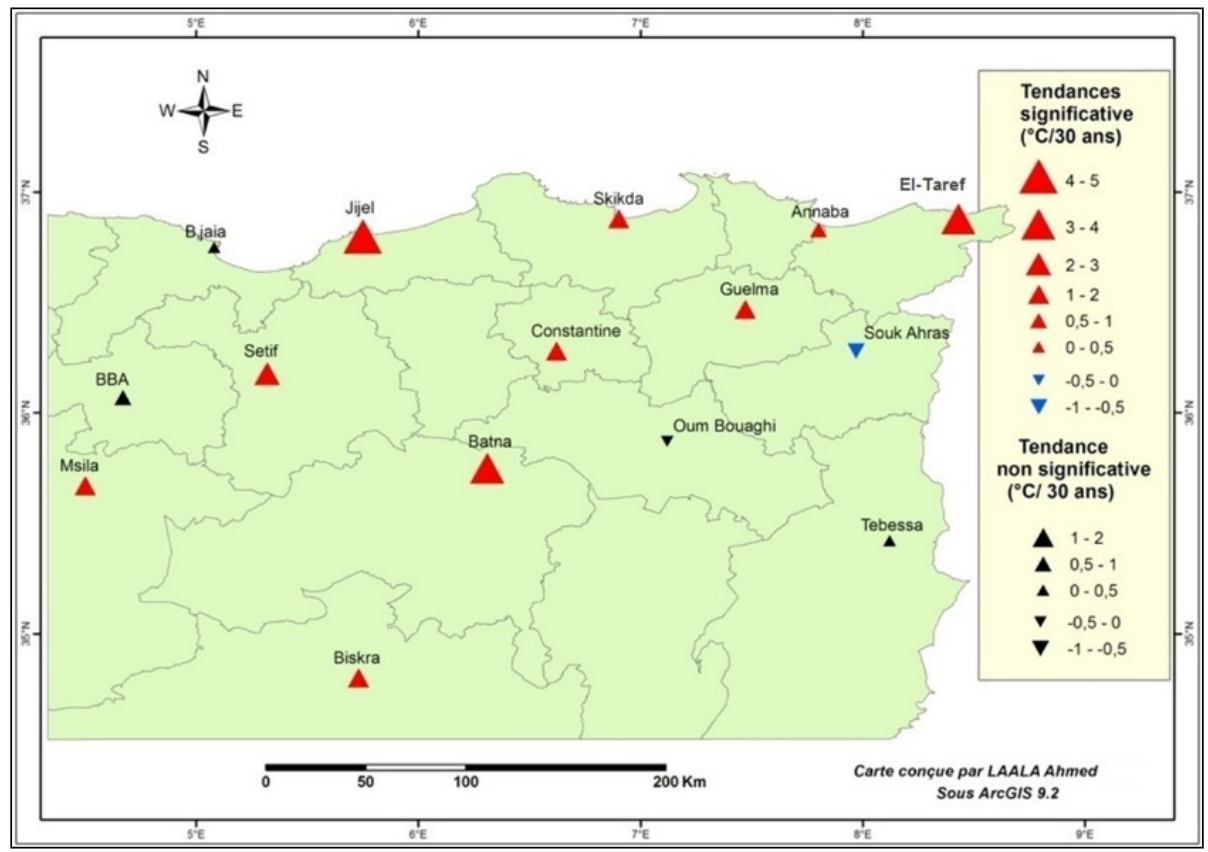

Fig.7: Tendances des amplitudes thermiques annuelles (1982-2011)

\section{Tendances des températures saisonnières}

\section{A)- Température minimale saisonnière}

La figure $n^{\circ} 8$ illustre la cartographie des tendances des températures minimales saisonnières pour la période étudiée (1982-2011). On constate une forte variation de ces tendances, allant de $-2.7^{\circ} \mathrm{C} / 30$ ans à plus de $2.5^{\circ} \mathrm{C} / 30$ ans. Les tendances non significatives sont affichées au niveau des sept wilayas dont la wilaya de Batna et M'sila restent toujours non significative pour les quatre saisons de l'année.

Soulignons également que la wilaya de Jijel affiche durant les quatre saisons de l'année une tendance significative à la baisse des températures minimales saisonnières. Alors que les wilayas de Bordj Bou-Arreridj et Tébessa montrent toujours des tendances significatives à la hausse. Le reste des wilayas marquent une alternance entre les tendances significatives et non significatives à travers les saisons.

Le refroidissement est très marqué en période hivernale et automnale (notamment pour la wilaya de Jijel) où les températures minimales diminuent globalement de $-2.5^{\circ} \mathrm{C} / 30$ ans. Cette diminution semble liée à l'année 2005, plutôt plus froide et marquée par une abondance des valeurs négatives durant la saison hivernale. 


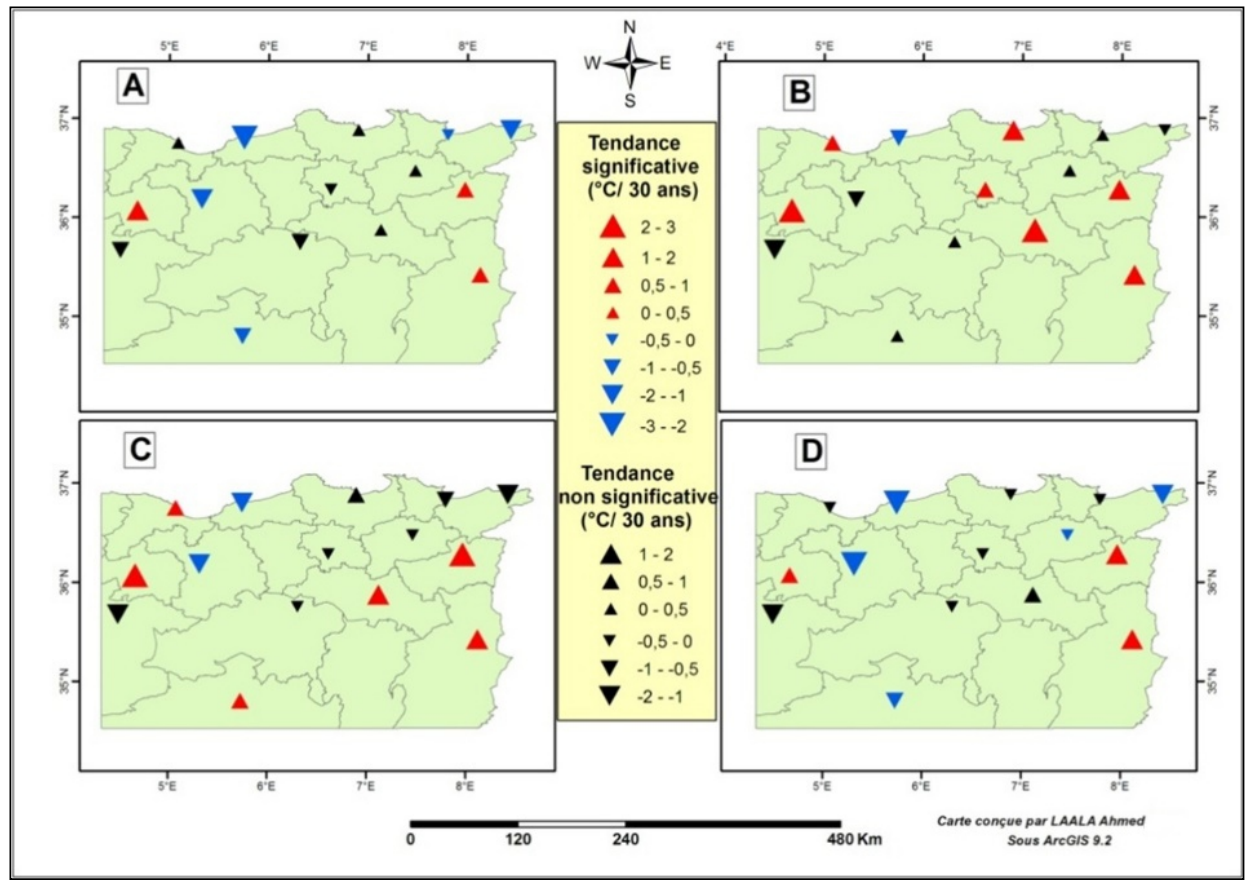

Fig.8: Tendances des températures minimales de la saison : hivernale (A), printanière (B), estivale (C) et automnale (D)

Le réchauffement est légèrement plus marqué en période printanière où les températures minimales des wilayas de Skikda Tébessa, Souk-ahras, Oum El-Bouagi et Bordj Bou-Arreridj gagnent globalement de $1^{\circ} \mathrm{C} / 30$ ans à plus de $2.5^{\circ} \mathrm{C} / 30$ ans. Cependant, le refroidissement est observé au niveau de cinq wilayas durant la saison hivernale, une wilaya pendant le printemps, deux wilayas durant l'été et cinq wilayas durant l'automne (fig.8).

\section{B)- Température maximale saisonnière}

L'analyse de la figure $n^{\circ} 9$ montre que nombre des wilayas qui affichent des tendances non significatives est plus élevé durant l'automne par rapport aux autres saisons de l’année. 


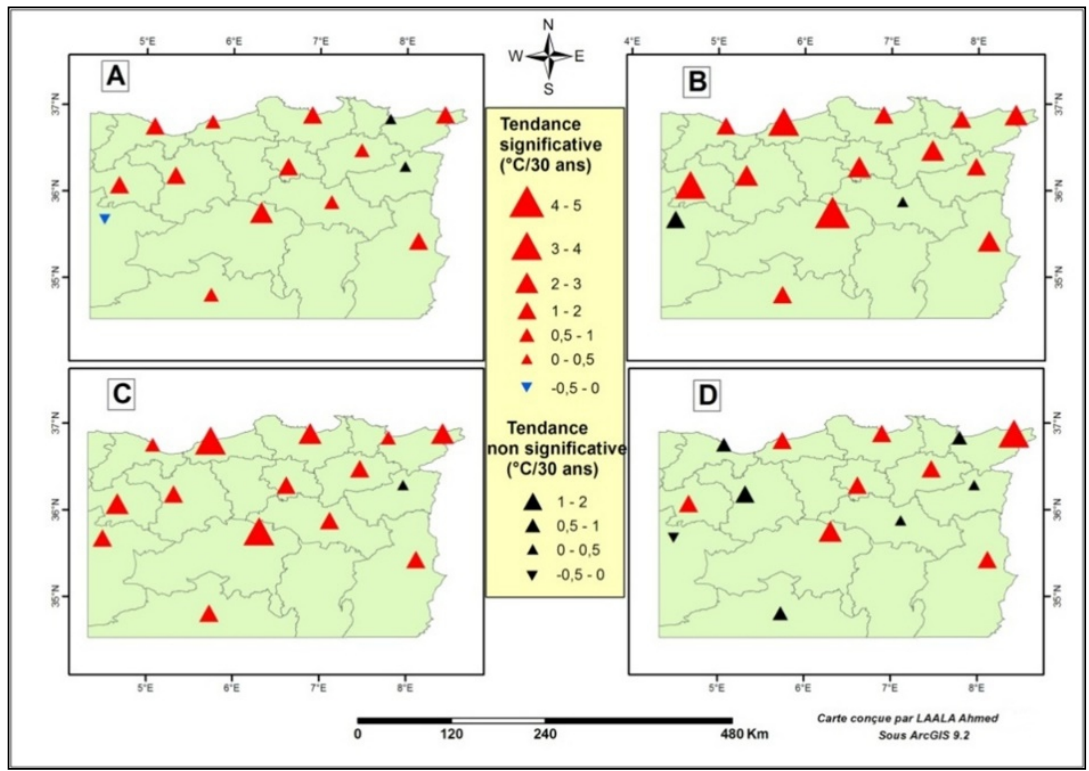

Fig9 : Tendances des températures maximales de la saison : hivernale (A), printanière (B), estivale (C) et automnale (D)

Toutefois, nous avons constaté que les températures maximales sont à la hausse pour toutes les wilayas sauf pour M'sila qui affiche une tendance négative, significative pour l'hiver et non significative au seuil de $5 \%$ pour l'automne. Le réchauffement le plus important est observé durant le printemps dans la wilaya de Batna $\left(4.1^{\circ} \mathrm{C} / 30 \mathrm{ans}\right)$ et durant l'été pour la wilaya de Jijel $\left(3.5^{\circ} \mathrm{C} / 30\right.$ ans). La hausse des températures maximales de la saison estivale est plus de $0,5^{\circ} \mathrm{C}$ dans les wilayas d'Annaba et Bejaia alors qu'elle se situe entre $3.2^{\circ} \mathrm{C}$ et $3,5^{\circ} \mathrm{C}$ pour les wilayas de Batna et Jijel. Ainsi, les tendances des températures maximales pour la saison estivale sont significatives au seuil de $5 \%$ pour toutes les wilayas sauf celle de SoukAhras (fig.9).

Par une simple comparaison entre la figure $n^{\circ} 1$ et 2 , il ressort que les températures maximales saisonnières ont connu une augmentation plus prononcée que les températures minimales saisonniers au cours de la période 1982 à 2011 et que le nombre des wilayas ayant des valeurs de tendance significatives au seuil de 5\% pour les températures maximales est plus grand que pour les températures minimales.

\section{C)- Amplitudes thermiques saisonnières}

L'examen de la carte ci-dessous montre que les tendances non significatives de ce paramètre sont observées au niveau des cinq wilayas durant l'hiver, quatre durant l'automne et trois durant le printemps et l'été. La saison estivale présente des tendances très importantes : $5.2^{\circ} \mathrm{C} / 30$ ans au 
niveau de la wilaya de Jijel, $3.6^{\circ} \mathrm{C} / 30$ ans dans la wilaya d'El-Taref, suivie de la saison automnale avec des tendances de l'ordre de $4^{\circ} \mathrm{C}$. La wilaya de Souk-Ahras présente toujours une tendance à la baisse de ce paramètre, significative pour l'été et l'automne et non significatif pour l'hiver et le printemps. Ainsi, Bejaïa affiche des tendances positives durant l'hiver, le printemps et l'automne et une tendance négative pour l'été.

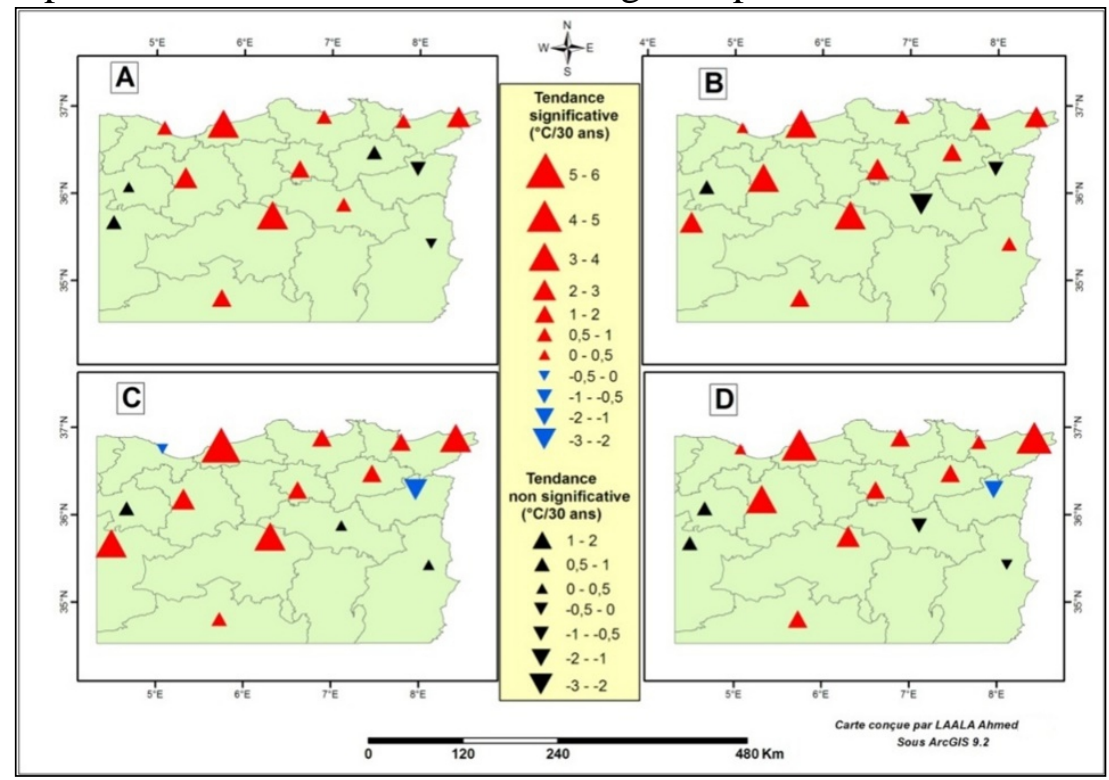

Fig10 : Tendances des amplitudes thermiques de la saison : hivernale (A), printanière (B), estivale (C) et automnale (D)

\section{Discussion}

A l'échelle régionale, une remarquable hausse des températures maximales annuelles de l'ordre de $3^{\circ} \mathrm{C} / 30$ ans est enregistrée à la wilaya de Batna, soit près de deux fois le taux de réchauffement de la wilaya de Sétif. Ce résultat est en accord avec le travail de Tabet-Aoul (2010) qui montre qu'en Algérie la hausse des températures au XXème siècle s'est située entre $1,5^{\circ}$ et $2^{\circ} \mathrm{C}$, soit plus du double de la hausse moyenne planétaire.

Nos résultats concordent aussi avec plusieurs études qui démontrent, au moyen des principaux postes climatologiques mondiaux, qu'il existe un réchauffement planétaire et envisage que la température moyenne de la terre aurait augmenté de $0,7^{\circ} \mathrm{C}$ en un siècle (Boden et al., 1994 ; Kelly et al., 1985 ; Jones et al., 1994 ; Mann et al., (1998) ; Wilson et Hansen, 1994 ; Vinnikov et al., 1994).

Pour les régions méditerranéennes, de nombreuses recherches sur les variations climatiques et particulièrement sur la tendance des températures ont été conduites au cours des dernières décennies. En France, les températures suivent la même tendance au cours des dernières années que 
celle relevée à l’échelle mondiale (Seguin, 2007). Dessens et Barés (1996) ont mis en évidence une forte augmentation de la température minimale notamment en été $\left(+0,93 \mathrm{C}^{\circ}\right)$ et en automne $\left(+0,88 \mathrm{C}^{\circ}\right)$ et cela à partir de l'analyse des longues séries des températures de la période 1910-1986. La même remarque a été signalé pour les température moyenne au cours du travail de Moisselin et al., 2002. Ces auteurs affirment une augmentation de ces températures de l'ordre de $0.9^{\circ} \mathrm{C}$ au cours du $\mathrm{XX}^{\mathrm{ème}}$ siècle, mais avec une nette accélération sur la dernière décennie de ce siècle et sur la première du $\mathrm{XXI}^{\text {ème }}$. Cette tendance est plus importante pour les températures minimales $\left(0.7\right.$ à $1.7^{\circ} \mathrm{C} /$ siècle $)$ que pour les maximales $\left(0\right.$ à $\left.1.3^{\circ} \mathrm{C}\right)$.

Ainsi, Fratianni et al., (2009) ont étudié les tendances des température de l'Italie et montre aussi une augmentation des températures saisonnières de l'ordre $0,07{ }^{\circ} \mathrm{C}$ pou les maximas et de $0,03{ }^{\circ} \mathrm{C}$ pour les minimas et cela durant la période de 1971-2000.

Concernant l'Espagne, l'analyse statistique des longues séries de température réalisée par Quereda et al., (2005) montre une tendance significative au réchauffement, avec une augmentation de la température moyenne annuelle comprise entre $0,5{ }^{\circ} \mathrm{C}$ et $1,2{ }^{\circ} \mathrm{C}$ pour la période 1870 1996. Cependant, les différentes valeurs de la tendance suggèrent que le réchauffement n’a pas affecté les stations de façon homogène.

Pour notre étude, les maximales tendent à une augmentation plus remarquable que les minimales et l'amplitude thermique est notable pour la majorité des wilayas étudiées. Ceci est en désaccord avec l'étude d'Easterling et al., (1997) qui montre que l'augmentation de la température minimale avait été supérieure à celle de la température maximale entraînant une diminution de l'amplitude thermique diurne.

Le résultat saisonnier révele aussi une tendance positive des températures maximales saisonnières pour presque toutes les wilayas étudiées avec bien sûre une certaines variabilité inter-saisonnière, ce qui montre la vigueur de l'accroissement des températures maximales au cours de la période 1982-2011. Cependant les températures minimales saisonnières présentent un mélange de baisse et de hausse pour les 15 wilayas étudiées.

En printemps et en été, les tendances significativement positives sont largement majoritaires, alors qu'en hiver et en automne, elles sont observées uniquement au niveau de trois stations météorologiques des wilayas de Tébessa, Souk-Ahras et Bordj Bou-Arreridj. Tabet-Aoul (2008) signale également une hausse de température moyenne de l'Algérie au cours des saisons d'hiver et d'automne durant la période (1930-2000) et une hausse nette des températures minimales et maximales sur le Nord du pays.

La distribution géographique des tendances des températures (minimales et maximales) pour la saison printanière est presque comparable à celle de la saison estivale, alors que celle de la saison automnale est 
pratiquement analogue à celle de la saison hivernale. Ceci nous permet de diviser l'année en deux saisons distinctes : une saison froide et une saison chaude (caractéristiques du climat méditerranéen).

\section{Conclusion}

L’analyse des séries temporelles des données thermiques a permis de mettre en évidence une variabilité spatio-temporelle des températures au niveau de l'Algérie orientale. Il témoigne d'un réchauffement évident au niveau de la majorité des wilayas étudiées.

Plus de la moitié de la période étudiée (1982-2011) est considérée comme chaude où le poids thermique le plus important est observé au niveau de la wilaya de Batna $\left(+15.8^{\circ} \mathrm{C}\right)$. Les dernières années de la période d'étude symbolisent les années chaudes pour la quasi-totalité des wilayas de l'Est algérien où l'anomalie des températures maximales est toujours positive. Il ressort aussi de cette étude que l'année 1994 est une année chaude pour toutes les wilayas étudiées.

Les résultats de ce travail constituent un cri d’alarme lancé aux décideurs pour définir des politiques stratégiques d’adaptation durables, puisque ces tendances vers le réchauffement auront toujours des incidences nocives et palpables sur les écosystèmes forestières. La liste de ces conséquences possibles qui en découlent est longue. Parmi eux, on peut citer : le débourrement et la floraison plus précoce des arbres, la chute plus tardive de leurs feuilles, avortements floraux, sensibilité au gel accrue, phénologie erratique, maturité échelonnée, risques accrues aux gels hivernaux et printaniers, difficulté de régénération, croissance limitée, forte vulnérabilité aux attaques massives des insectes...etc. Ce réchauffement peut provoquer aussi un déplacement des aires de répartition de la majorité des essences forestières vers des habitats favorable thermiquement à leurs croissances et leurs développements. Il faut cependant signaler que la réaction et le déplacement de ces écosystèmes n’est pas immédiate.

\section{References:}

Berry, J., \& Bjorkman, O. (1980). Photosynthetic response and adaptation to temperature in higher plants. Ann Rev Plant Phys 31, 491-543

Boden, T. A., Kaiser, D. P., Sepanski, R. J., \& Stoss F. W. (1994). Trends 93: A compendium of data on global change ORNL/CDIAC-65. Carbon Dioxide Information Analysis Center, Oak Ridge National Laboratory, Oak Ridge, Tennessee, États-Unis, 984 p.

Dajoz, R. (1985). Précis d’écologie. Ed. Bordas, Paris, 5ème édition, 505p

Davis, M. B. (1989). Retards dans la réponse de la végétation au réchauffement climatique. Changement climatique 15:75-82. 
Dessens, J., \& Barés, A. (1996). Evolution of the maximum and minimum temperatures in France from 1891-1992, J. Meteorol. 21 (1996) 41-48.

Easterling, D. R., Horton, B., Jones, P. D., Peterson, T.C., Karl, T. R., Parker, D. E., Salinger, M. J., Razuvayev, V., Plummer, N., Jamason, P., Folland, C. K.(1997). Maximum and minimum temperature trends for the globe, Science 277 (1997) 364-367.

Fratianni, S., Brunatti, S., Acquaotta, F., Terzago, S. (2009). Tendance de températures et précipitations neigeuses en vallee Maira (Piemont SudOccidentale, Italie). Geographia Technica. Numéro spécial, 2009, 187-192 GIEC, (2007). Bilan 2007 des changement climatiques. Contribution des groupes de travail I, II et III au Quatrième d'évaluation du Groupe Intergouvernemental sur 1'Evolution du Climat - GIEC, 103p.

Hamann, A., \& Wang, T. (2006). Potential effects of climate change on ecosystem and tree species distribution in British Columbia. Ecology 87: 2773-2786.

Havaux, M., \& Lannoye, R. (1985). Effets des basses températures positives sur les réactions photochimiques primaires de la photosynthèse du maïs (Zea mays L., cv. « LG 9 »). Agronomie, 1985, 5 (4), 331-337.

Heller, R., Esnault, R., \& Clance, C. (1989). Physiologie végétale. Nutrition. Tom1. 4ème édition, p201

Jones, P. D., Wigley, T. M. L., \& Briffa, K. R. (1994). Global and hemispheric temperature anomalies-land and marine instrumental records, in Boden T. A., D. P., Kaiser, R. J. Sepanski et F. W. Stoss (eds). Trends 93: A compendium of data on global change. ORNL/CDIAC-65. Carbon Dioxide Information Analysis Center, Oak Ridge National Laboratory, Oak Ridge, Tennessee, États-Unis, 603-608

Kelly, P. M., Jones, P. D., Wigley, T. M. L., Bradley, R. S., Diaz, H. F. \& Goodess, C. (1985). The extended Northern Hemisphere surface air temperature record: 1851-1984. Preprints, Third Conference on Climate Variations and Symposium on Contemporary Climate: 1850-2100, American Meteorological Society, Boston, États-Unis, 23-24.

Kendall, M. G. (1975). Rank Correlation Methods, Charles Griffen, London,156p

King, A. W., Gunderson, C, A., Post, W. M., Weston, D. J., Wulschleger, S. D. (2006). Plant respiration in a warmer world. Science, 302, 36-37

Mann, H. B. (1945). Non Parametric Test Against Trend. Econometrika, 13, 245-259.

Mann, M. E., Bradley, R.S., Hughes, M. K. (1998). Global-scale temperature patterns and climate forcing over the past six centuries, Nature 392 (1998) 779-787. 
McKenney, D.W., Pedlar, J. H., Lawrence, K., Campbell., K, Hutchinson, M. F. (2007). Potential impacts of climate change on the distribution of North American trees. Bioscience 57: 939-948.

Merle, J. (1980). Océan et climat. Les fonctions thermiques de l’océan dans la dynamique du climat. Trav, Doc. OSTROM. La météorologie, VIe série n²2 septembre 1980. 87, 85-95.

Méthy, M., Trabaud, L. (1990). Caractérisation de la résistance au froid de deux espèces sclérophylles méditerranéennes par la fluorescence de la chlorophylle. Ann.Sci.For., 1990, 47, 141-148.

Moisselin, J. M., Schneider, M., Canellas, C., \& Mestre, O. (2002). « Les changements climatiques en France au XXe siècle : étude des longues séries homogénéisées de température et de précipitations », La Météorologie, 38, 45-56

Myneni, R. B., Keeling, C.D., Tucker, C.J., Asrar, G., Nemani, R. R. (1997). Increased plant growth in the northern high latitudes from 1981 to 1991. Nature 386: 698-702

Quereda, J. S., Gil, A. O., Olcina, J. C., Rico, A. A., Montón, E. C., \& Rabourdin, S. (2005): Changement climatique. Comprendre et agir. Delachaux et Niestlé, Paris, 286 p.

Seguin, B. (2007). Les changements climatiques et les impacts observés sur les écosystèmes terrestres, RDV techniques hors-série ${ }^{\circ} 3$, pp. 3-8

Tabet-Aoul, M. (2008). « Impacts du changement climatique sur les agricultures et les ressources hydriques au Maghreb ». Note d'alerte du CIHEAM, n48.

Tabet-Aoul, M. (2010). Développement et environnement au Maghreb : contraintes et enjeux, pp. 66-70.

Vinnikov, K. Y., Groisman, P. Y., \& Lugina, K. M. (1994). Global and hemispheric temperature anomalies from instrumental surface air temperature records, in Boden T. A., D. P. Kaiser, R. J. Sepanski et F. W. Stoss (eds). Trends 93: A compendium of data on global change. ORNL/CDIAC-65. Carbon Dioxide Information Analysis Center, Oak Ridge National Laboratory, Oak Ridge, Tennessee, États-Unis, 615-627.

Wilson, H., \& Hansen, J. (1994). Global and hemispheric temperature anomalies from instrumental surface air temperature records, in Boden T. A., Kaiser, D. P., Sepanski, R. J., et Stoss, F. W. Trends 93: A compendium of data on global change. ORNL/CDIAC- 65. Carbon Dioxide Information Analysis Center, Oak Ridge National Laboratory, Oak Ridge, Tennessee, États-Unis, 609-614. 